\title{
Identification of synaptic connections in neural ensembles by graphical models
}

\author{
Rainer Dahlhaus ${ }^{\mathrm{a}, *}$, Michael Eichler ${ }^{\mathrm{a}}$, Jürgen Sandkühler ${ }^{\mathrm{b}}$ \\ a Institut für Angewandte Mathematik, Universität Heidelberg, Im Neuenheimer Feld 294, D-69120 Heidelberg, Germany \\ ${ }^{\mathrm{b}}$ II. Physiologisches Institut, Universität Heidelberg, Im Neuenheimer Feld 326, D-69120 Heidelberg, Germany
}

Received 25 March 1997; received in revised form 23 May 1997; accepted 26 May 1997

\begin{abstract}
A method for the identification of direct synaptic connections in a larger neural net is presented. It is based on a conditional correlation graph for multivariate point processes. The connections are identified via the partial spectral coherence of two neurons, given all others. It is shown how these coherences can be calculated by inversion of the spectral density matrix. In simulations with GENESIS, we discuss the relevance of the method for identifying different neural ensembles including an excitatory feedback loop and networks with lateral inhibitions. (C) 1997 Elsevier Science B.V.
\end{abstract}

Keywords: Neuronal net; Synaptic connectivity; Multivariate point processes; Partial spectral coherence; Graphical models

\section{Introduction}

The cellular and molecular mechanisms of signal transduction in the nervous system have been studied intensively and, as a consequence, a better knowledge and understanding of some basic processes of information handling by neurons has been achieved (Trimble et al., 1991; Bliss and Collingridge, 1993; Wickmann and Clapham, 1995; Schuldiner et al., 1995). In contrast, little is known about the organization and function of complex neuronal networks. Experimental methods are now available to simultaneously monitor electrical activity of a large number of neurons in real time. This may be achieved by multiple single neuron recordings (Krüger, 1983) or by use of voltage sensitive dyes (Grinvald et al., 1988; Parsons et al., 1991). The use of four closely spaced electrodes ('tetrode') allows identification of discharges of ten to 15 individual neurons. This is a considerably higher yield than that obtained by two electrodes ('stereotrode') or the conventional

* Corresponding author. Tel.: + 496221 544989; fax: + 496221 545331. single microelectrode recordings (Gray et al., 1995). Commonly used cross-correlation analysis may detect temporally correlated discharges (Hata et al., 1991; Alloway et al., 1993), which may be relevant for assembly coding of information (Lindsay et al., 1992; Singer, 1993). The interpretation of data is, however, hampered by the fact that cross-correlograms may not reveal the nature of the correlations, i.e. it is not possible to differentiate between direct and indirect connections and a common input to the neurons under study.

The aim of this paper is to present a method for such an identification of synaptic connections in a larger neural net based on frequency domain methods.

Brillinger et al. (1976) have used the partial spectral coherence for point processes as a tool for identifying synaptic interactions between three cells. They used this method to discriminate between direct and indirect connections between the neurons. In Rosenberg et al. (1989), the method has been used to distinguish whether two neurons interact directly or are influenced by common input of one or more cells. Earlier, Gersch (1972) had used the partial spectral coherence for time series to study causality for three-dimensional time 
series. In Gersch and Goddard (1970), the method has been applied to locate the site of an epileptic seizure in the cat brain. Kamiński and Blinowska (1991) used frequency domain methods based on a parametric time series model to distinguish between direct and indirect transfer of information in a multichannel EEG process.

In the present paper, we extend the method of identification by the partial spectral coherence to a larger net of neurons and combine it with recent results on graphical models for multivariate data (Whittaker, 1990; Lauritzen, 1996; Cox and Wermuth, 1996). The theoretical framework of our method is the theory of stationary point processes. In Section 2, we present the method and discuss its properties. In Section 3, we study the performance of the proposed method by applying it to various neural nets generated with the GENESIS-simulation program (Bower and Beeman, 1995).

Alternatively, one could try to use time domain methods for this identification. Gerstein and Aertsen (1985) have introduced the gravity method where the neurons are represented by particles with 'charges' related to the spike trains of the neurons. The resulting forces on the particles cause aggregation of those neurons which exhibit correlated firing.

\section{The identification method}

The method proposed in this paper for the identification of synaptic connections is based on a stochastic graphical interaction model for multivariate point processes. This model is new as well and could be applied also to other type of data.

As explained in more detail in Section 2.4 the vertices in the graph represent the components of the point process (the different neurons) while an edge between two vertices reflects a 'direct' connection between the two corresponding neurons. Thus, we can distinguish between direct connections and indirect connections via other neurons. More precisely, an edge reflects a partial correlation between the two components and some modification is necessary to conclude to a physically existing connection. This modification procedure is explained in Section 2.5.

The conditional correlation graph is estimated by using the partial spectral coherence of two components of the point process given all other components. The phase of the partial spectrum gives some additional information on the time delay of the signals and therefore also on the direction of the connections. This methodology is presented in Sections 2.1, 2.2 and 2.3 while the graphical model itself is introduced in Section 2.4 .

The fitted graph therefore is based on a stochastic model and estimated from the measurements at hand.
Two problems have to be investigated: first, the question whether the proposed estimation method is reasonable within the stochastic model, i.e. whether the graph of a multivariate point process which reflects certain probabilistic properties of the process can be identified from a realization of the point process with the proposed method; and second, whether the stochastic graphical model is a reasonable model for the identification of physically existing synaptic connections in neural ensembles. Both questions are investigated via simulation studies in Section 3.

\subsection{Multivariate point processes}

The method discussed in this paper is based entirely on the relation between the sequences of the times of occurrence of action potentials in the recorded spike trains of different neurons. Therefore, the spike trains of a group of neurons can be represented as a multivariate stochastic point process. A point process in general refers to an ordered sequence of isolated events $\left\{\sigma_{j}\right\}_{j \in \mathbb{Z}}$ occurring randomly in time. Such a point process associated with the spike train of neuron $A$ can be described by the counting process $N_{A}(t)$, where $N_{A}(t)$ is the total number of action potentials up to time $t$ of neuron $A$.

Subsequently, it will be assumed that the correlation structure of the multivariate point process does not change with time (the process is second-order stationary) and that events widely separated in time occur essentially independently (the process is mixing). As nerve cell action potentials cannot occur simultaneously, the associated point process is also orderly, i.e. there are no multiple events.

Parameters of interest in the analysis of spike trains are the mean-intensity of process $N_{A}$, defined by

$p_{A}=\lim _{h \downarrow 0} \operatorname{Prob}\{A$ spike in $(t, t+h]\} / h$

and the cross-product density at lag $u$ between processes $N_{A}$ and $N_{B}$, defined by

$$
\begin{aligned}
& p_{A B}(u) \\
& =\lim _{h, h^{\prime} \downarrow 0} \operatorname{Prob}\{A \text { spike in }(t+u, t+u+h] \\
& \left.\quad \text { and } B \text { spike in }\left(t, t+h^{\prime}\right]\right\} / h h^{\prime} .
\end{aligned}
$$

In the case $A=B$, the product density $p_{A A}(u)$ is defined in such a way that it is continuous at $u=0$. Alternatively, one might consider the cross-intensity function,

$$
\begin{aligned}
& m_{A B}(u) \\
= & \lim _{h \downarrow 0} \operatorname{Prob}\{A \text { spike in }(t+u, t+u+h] \mid B \text { spike at } t\} \\
& / h,
\end{aligned}
$$

which is related to the cross-product density by $m_{A B}(u)=p_{A B}(u) / p_{B}$, or the cross-covariance density, $q_{A B}(u)=p_{A B}(u)-p_{A} p_{B}$. 


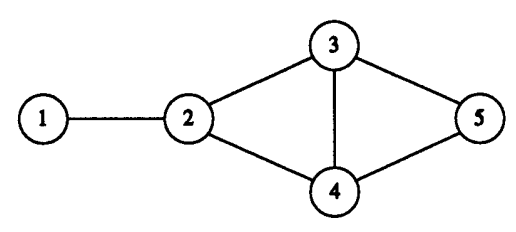

Fig. 1. A simple graph.

In the frequency domain, the corresponding secondorder parameters are the cross-spectrum between processes $N_{A}$ and $N_{B}$,

$f_{A B}(\lambda)=\frac{1}{2 \pi} \int_{-\infty}^{\infty}\left(p_{A B}(u)-p_{A} p_{B}\right) \exp (-i \lambda u) \mathrm{d} u$

and the auto-spectrum of process $N_{A}$,

$f_{A A}(\lambda)=\frac{p_{A}}{2 \pi}+\frac{1}{2 \pi} \int_{-\infty}^{\infty}\left(p_{A A}(u)-p_{A}^{2}\right) \exp (-i \lambda u) \mathrm{d} u$

More generally, if $A$ and $B$ are sets consisting of one or more neurons, we denote by $f_{A B}(\lambda)$ the matrix with elements $f_{a b}(\lambda)$ where $a \in A$ and $b \in B$.

Suppose the spike trains of $K$ different neurons have been recorded over a time interval of length $T$. Then the above time domain parameters can be estimated by the empirical intensity

$\hat{p}_{A}^{(T)}=\frac{N_{A}(T)}{T}$

and by the cross-correlation histogram

$\hat{m}_{A B}^{(T)}(u)=\frac{1}{b N_{B}(T)} \#\left\{u-\frac{b}{2}<\sigma_{j}-\tau_{k}<u+\frac{b}{2} ; \tau_{k} \neq \sigma_{j}\right.$,

$j, k=1,2, \ldots\}$,

where '\#' stands for 'the number of', $b$ denotes a bin width and $\left\{\sigma_{j}\right\}$ and $\left\{\tau_{k}\right\}$ denote the observed times of $A$ spikes and $B$ spikes, respectively.

The approach in the frequency domain is based on the finite Fourier transforms of the individual spike trains, which are given by

$d_{A}^{(T)}(\lambda)=\sum_{0<\sigma_{j} \leq T} h\left(\sigma_{j} / T\right) \exp \left(-i \lambda \sigma_{j}\right)$,

where $\left\{\sigma_{j}\right\}$ are the observed spike times for neuron $A$ and $h(\cdot)$ is a data taper, by which the convergence properties of the spectral estimates can be improved (Brillinger, 1981). The Fourier transform $d_{A}^{(T)}$ can be computed efficiently by using a fast Fourier transform

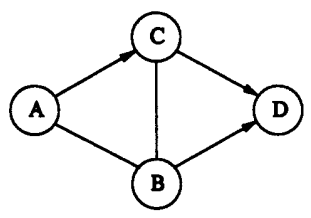

Fig. 2. Identifying the directed graph. algorithm based on a discrete approximation of the point process $N_{A}$ (Rigas, 1993). The spectral density matrix $f(\lambda)=\left(f_{A B}(\lambda)\right)_{A, B=1, \ldots, K}$ then is typically estimated componentwise by

$\hat{f}_{A B}(\lambda)$

$=\frac{1}{2 \pi H_{2} T} \sum_{s \in \mathbb{Z}} w_{T}\left(\lambda-\frac{2 \pi s}{T}\right) d_{A}^{(T)}\left(\frac{2 \pi s}{T}\right) d_{B}^{(T)}\left(-\frac{2 \pi s}{T}\right)$,

where $H_{2}=\int_{0}^{1} h(t)^{2} \mathrm{~d} t$ and $w_{T}(\lambda)=w(\lambda / T) / T$ for some kernel function $w(\cdot)$ with bounded support (Brillinger, 1981). A more detailed discussion of frequency methods may be found in Brillinger et al. (1976); Rosenberg et al. (1989).

The cross-spectrum $f_{A B}(\lambda)$ measures the strength of association between two neurons $A$ and $B$ : if the crossspectrum vanishes for all frequencies, the two spike trains $N_{A}$ and $N_{B}$ are linearly independent. A reasonably rescaled version of the cross-spectrum is the spectral coherence $\left|R_{A B}(\lambda)\right|^{2}$ where

$R_{A B}(\lambda)=\frac{f_{A B}(\lambda)}{\left(f_{A A}(\lambda) f_{B B}(\lambda)\right)^{1 / 2}}$.

Unlike the cross spectrum, the spectral coherence is bounded and takes values between zero and one. It satisfies

$\left|R_{A B}(\lambda)\right|^{2}=\lim _{T \rightarrow \infty}\left|\operatorname{corr}\left\{\mathrm{d}_{A}^{(T)}(\lambda), \mathrm{d}_{B}^{(T)}(\lambda)\right\}\right|^{2}$.

Therefore, if $\left|R_{A B}(\lambda)\right|^{2}$ differs from zero for some frequency component, this implies a correlation of the two spike trains $N_{A}$ and $N_{B}$ and thus some connection between the nerve cells $A$ and $B$. However, we are not able to decide on the basis of the spectral coherence whether the two neurons $A$ and $B$ are directly connected or only indirectly connected over one or more other neurons.

\subsection{The partial spectral coherence}

In order to decide whether two neurons $A$ and $B$ are connected directly or are influenced by a common input $C$, Brillinger et al. (1976) recommended to estimate the partial spectral coherence $\left|R_{A B \mid C}(\lambda)\right|^{2}$ where

$R_{A B \mid C}(\lambda)=\frac{f_{A B \mid C}(\lambda)}{\left(f_{A A \mid C}(\lambda) f_{B B \mid C}(\lambda)\right)^{1 / 2}}$

with

$f_{A B \mid C}(\lambda)=f_{A B}(\lambda)-f_{A C}(\lambda) f_{C C}(\lambda)^{-1} f_{C B}(\lambda)$

(see Rosenberg et al., 1989; Brillinger, 1981). Here the common input $C$ might consist of several neurons.

In contrast to the ordinary coherence, the partial spectral coherence measures the dependence between the processes $N_{A}$ and $N_{B}$ after the linear effects of the (multivariate) process $N_{C}$ have been removed (Rosen- 
(a)

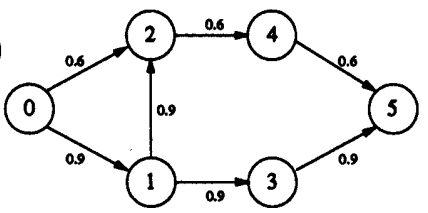

(b)

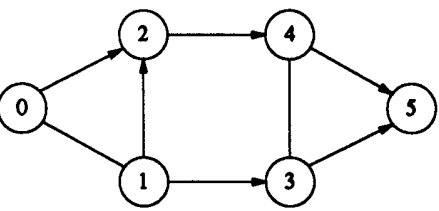

Fig. 3. (a) Real graph for the Hawkes model data with $\alpha_{A B} / \beta_{A B}$ given for each connection $\left(\beta_{A B}=500, u_{A B}=0.02\right)$. (b) Estimated conditional correlation graph with some directions derived from the phase spectrum.

berg et al., 1989). For example, consider the case of three neurons $A, B$, and $C$ where $A$ is connected with $C$ and $C$ is connected with $B$. Then the spectral coherency $\left|R_{A B}(\lambda)\right|^{2}$ differs from 0 for some frequencies $\lambda$, which shows the dependence between $A$ and $B$. If neural activity were purely driven by linear dynamics, then the partial spectral coherency $\left|R_{A B \mid C}(\lambda)\right|^{2}$ would vanish for all $\lambda$ indicating that the connection from $A$ to $B$ is not direct but via neuron $C$. However, there is evidence that neural activity cannot completely be described by linear dynamics. Nevertheless, the partial spectral coherence seems to work well for the discrimination between direct and indirect connections (Brillinger et al., 1976; Rosenberg et al., 1989).

In this paper, we use the partial spectral coherence $\left|R_{A B \mid C_{A B}}(\lambda)\right|^{2}$, where $\mathrm{C}_{A B}$ denotes the set of all neurons without neurons $A$ and $B$, to build a graph which reflects the correlation structure of a multivariate point process and, after some modification also the connections in a neural net. Conditioning on the set $C_{A B}$ turns out to be the key step to obtain a meaningful graph which is consistent with intuition (Section 2.4).

The partial spectral coherences, which are necessary to build the graph, can easily be computed by inversion of the spectral density matrix $f(\lambda)$ as it has been shown in Dahlhaus (1995) for ordinary time series. More precisely, let $g(\lambda)=f(\lambda)^{-1}$ be the inverse of the spectral matrix and

$d_{A B}(\lambda)=\frac{g_{A B}(\lambda)}{\left(\left(g_{A A}(\lambda) g_{B B}(\lambda)\right)^{1 / 2}\right.}$.

Then it can be shown as in Theorem 3.2 of Dahlhaus (1995) that

$d_{A B}(\lambda)=-R_{A B \mid C_{A B}}(\lambda)$

and

$g_{A A}(\lambda)=f_{A A \mid C_{A}}(\lambda)^{-1}$,

where $C_{A}$ consists of all neurons except neuron $A$.

Using the spectral density matrix with components estimated by Eq. (1) leads in the same way to the estimates $\hat{R}_{A B \mid C}(\lambda)$. These estimates are numerically identical to the estimates one would obtain by using (Eq. (2)) and (Eq. (3)) with the estimated spectra. However, the latter is much more time consuming since it requires at each $\lambda$ for the calculation of each of the $K(K-1) / 2$ partial coherences the inversion of a $(K-$
2) $\times(K-2)$ matrix while the former only requires at each $\lambda$ the inversion of one $K \times K$ matrix.

\subsection{The partial phase-spectrum}

When examining the structure of a neural net, we are interested not only in the strength of a connection, which we can measure by the partial coherence, but also in its direction, which is indicated by a time delay in the transmission of signals which is due to the propagation of action potentials along the axon of the sending neuron and the synaptic delay. Rosenberg et al. (1989) have shown that such a time delay in the relation between two spike trains $N_{A}$ and $N_{B}$ is related to the phase-spectrum $\phi_{A B}(\lambda)$ which is defined as the argument of the cross-spectrum $f_{A B}(\lambda)$. If, for example, the process $N_{2}$ is a shifted version of the process $N_{1}$, i.e. $N_{2}(t)=N_{1}\left(t+u_{0}\right)$, then we have $\phi_{21}(\lambda)-u_{0} \lambda$ and the delay $u_{0}$ can be determined from the slope of the phase-spectrum.

In the case where indirect connections may be present, Rosenberg et al. (1989) suggested to use the partial phase-spectrum $\phi_{A B \mid C_{A B}}(\lambda)$ which is defined as the argument of the partial cross-spectrum, that is

$\phi_{A B \mid C_{A B}}(\lambda)=\arg f_{A B \mid C_{A B}}(\lambda)=\arg R_{A B \mid C_{A B}}(\lambda)$.

To illustrate the difference to the ordinary phasespectrum, we consider independent processes $N_{1}(t)$ and $\varepsilon(t)$ and derived processes $N_{2}(t)=N_{1}\left(t+u_{1}\right)+\varepsilon(t)$ and $N_{3}(t)=N_{1}\left(t+u_{2}\right)+N_{2}\left(t+u_{3}\right)$. Here, the processes $N_{1}$ and $N_{3}$ are not only directly connected (with lag $u_{2}$ ) but also indirectly via $N_{2}$ (with lag $u_{1}+u_{3}$ ). Elementary manipulations now show that

$$
\begin{aligned}
& f_{31}(\lambda)=\left(\mathrm{e}^{-i \lambda u_{2}}+\mathrm{e}^{-i \lambda\left(u_{1}+u_{3}\right)}\right) f_{11}(\lambda) \\
& =\mathrm{e}^{-i \lambda\left(u_{2}+\left(u_{1}+u_{3}\right)\right) / 2} \cdot 2 \cos \left(-\lambda\left(u_{2}+\left(u_{1}+u_{3}\right)\right) / 2\right) f_{11}(\lambda)
\end{aligned}
$$

and by (Eq. (3)) with $f_{21}(\lambda)=\mathrm{e}^{-i \lambda u_{1}} f_{11}(\lambda)$ and

$f_{32}(\lambda)=\mathrm{e}^{-i \lambda\left(u_{2}-u_{1}\right)} f_{11}(\lambda)+\mathrm{e}^{-i \lambda u_{3}} f_{22}(\lambda)$

$f_{31 \mid 2}(\lambda)=\mathrm{e}^{-i \lambda u_{2}}\left(f_{11}(\lambda)-f_{11}(\lambda)^{2} / f_{22}(\lambda)\right)$,

which implies

$\phi_{31}(\lambda)=-\lambda\left(u_{2}+\left(u_{1}+u_{3}\right)\right) / 2$

and

$\phi_{31 \mid 2}(\lambda)=-\lambda u_{2}$. 


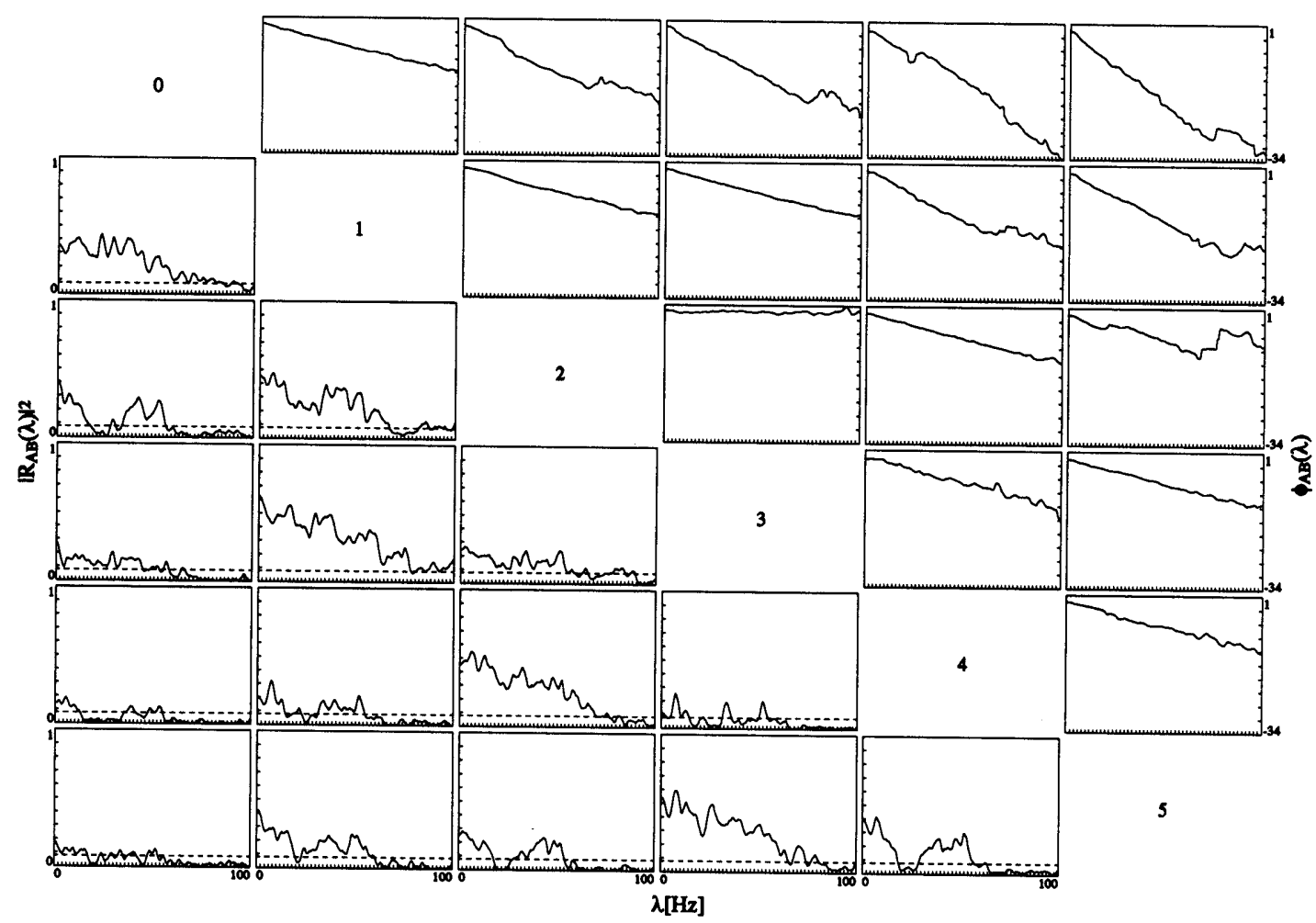

Fig. 4. Estimated coherences $\mid R_{A B}(\lambda)^{2}$ and phase spectra $\phi_{A B}(\lambda)$ for the Hawkes model data. The horizontal dashed lines represent a $95 \%$ test bound for the supremum of all coherence under the assumption that the two are uncorrelated.

The partial phase $\phi_{31 \mid 2}(\lambda)$ is the phase one would expect if no indirect connection were present, whereas the ordinary phase $\phi_{31}(\lambda)$ yields an average of the delays of direct and indirect connections.

Eq. (4) suggests to fit a linear regression model to the partial phase curves and to estimate the delay as the slope of the least squares line. As the variance of the partial phase depends on the partial coherence and therefore is not constant, one has to use a weighted least squares procedure as it has been described in Rosenberg et al. (1989). We mention, that these weights are zero at those frequencies where the partial coherence is zero.

\subsection{Conditional correlation graphs for multivariate point processes}

We now show how the partial spectral coherence can be used to define a graph for the correlation structure of a multivariate point process. A rather detailed discussion in the context of multivariate time series has been given in Dahlhaus (1995). The situation for point processes is basically the same.

Suppose we observe a multivariate point process $N(t)$ with components $N_{A}(t)$, where $A=1, \ldots, K$. We identify the components with the vertices of a graph, i.e. we have the set of vertices $V=\{1, \ldots, K\}$. An edge between vertices $A$ and $B$ is defined to be missing if the processes
$N_{A}(t)$ and $N_{B}(t)$ are uncorrelated (at all lags) after the linear effects of all other components have been removed. This is the case if and only if the partial cross-spectrum $f_{A B \mid C_{A B}}(\lambda)$ as given in (Eq. (3)) is zero for all frequencies (Rosenberg et al., 1989). As discussed above (see also Dahlhaus, 1995, Theorem 3.2), this means that we have no edge in the conditional correlation graph between $A$ and $B$ if and only if the corresponding component in the inverse spectral matrix, $g_{A B}(\lambda)$ or equivalently $d_{A B}(\lambda)$, is zero for all frequencies.

Suppose, for example, that we have a five dimensional point process with conditional correlation graph as given by Fig. 1 . The processes $N_{1}$ and $N_{5}$ are then for example uncorrelated given the linear effect of the other neurons. Our intuition of the graph says that the dependence between $N_{1}$ and $N_{5}$ is 'via' $N_{2}$. Such intuitive interpretations of the graph can be made rigorous by a number of properties (Dahlhaus, 1995, proposition 14). For example, the 'separation theorem' says that whenever one variable (or a whole set of variables) 'separates' two other variables (or sets of variables) then these two are uncorrelated given the separating one. This theorem allows, for example, in the above graph the interpretation that if $N_{2}$ (or alternatively the set $\left\{N_{3}, N_{4}\right\}$ ) is removed, then $N_{1}$ and $N_{5}$ are uncorrelated while removing $N_{3}$ alone would not help. 


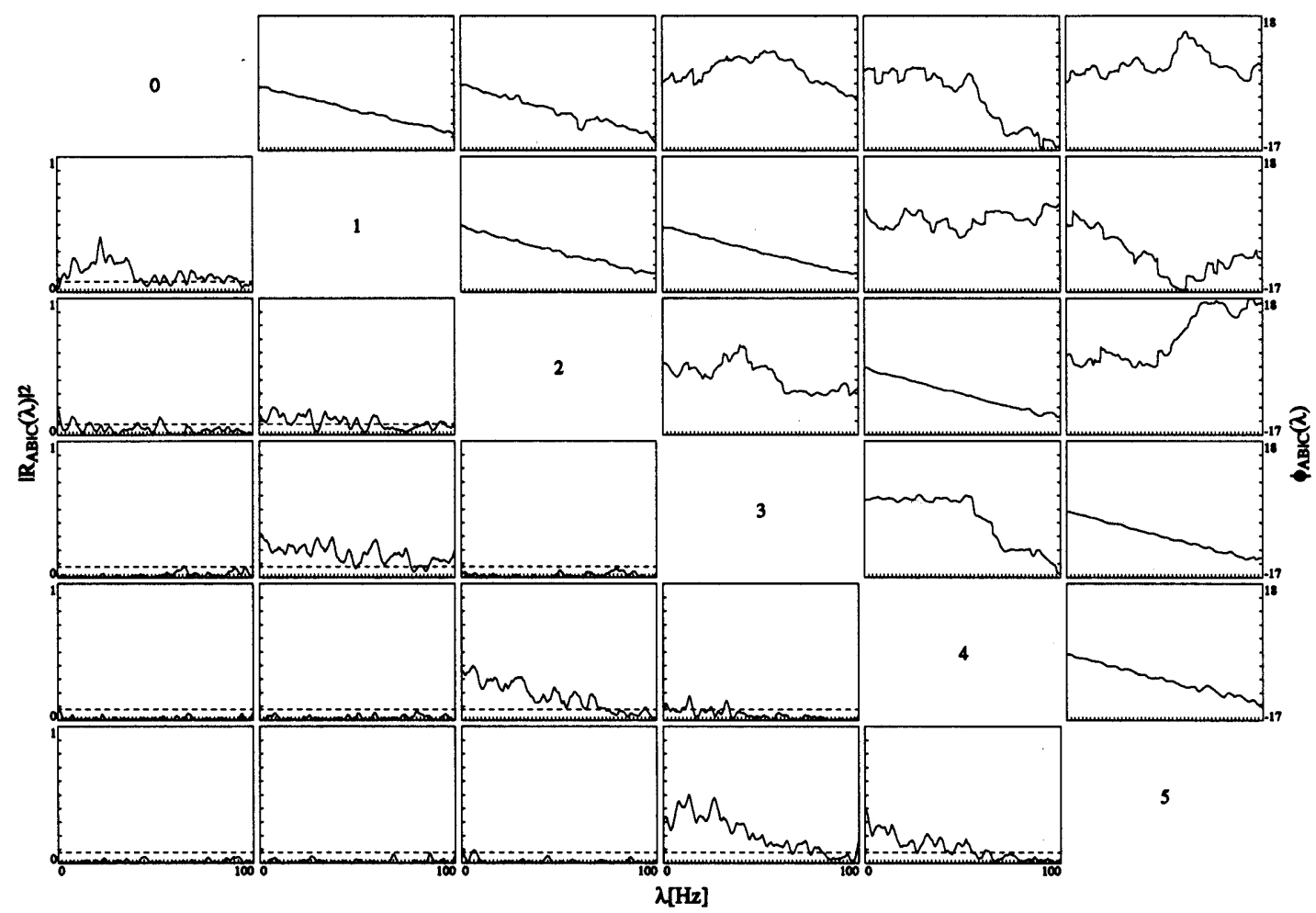

Fig. 5. Estimated coherences $\left|R_{A B \mid C_{A B}}(\lambda)\right|^{2}$ and partial phase spectra $\phi_{A B \mid C_{A B}}(\lambda)$ for the Hawkes model data. The horizontal dashed lines represent a $95 \%$ test bound for the supremum of all partial coherences under the assumption that the two processes are uncorrelated conditional on the other processes.

Although intuitively obvious, these properties are not easy to prove theoretically. However, their existence guarantees that certain probabilistic properties of the multivariate point process are in coincidence with the properties of the graph - thus making the conditional correlation graph of a point process a valuable tool for the visualization of its properties.

Given the observed point processes we use the empirical spectral coherence $\hat{R}_{A B \mid C_{A B}}(\lambda)$ with a test derived in Section 2.6 below for deciding whether the edge between $A$ and $B$ is present. The idea is to use the resulting estimate of the conditional correlation graph as the basis for an estimate of the graph reflecting the physically existing neural net.

It is not obvious whether these two graphs coincide and what the relationship between these graphs are. In the next section we investigate this question theoretically for a specific model of mutually exiting point processes. This model allows for the necessary theoretical calculations but is not fully adequate for neural nets since it neglects nonlinear dynamics such as refractory periods after the occurrence of a spike.

\subsection{The identification of directed acyclic graphs for mutually exciting point processes}

Hawkes (1971a,b) has introduced a model for mutu- ally exciting point processes. For such a process, the intensity vector at any time $t$ depends linearly on the history $H_{\mathrm{t}}$ of the process up to that instant. More precisely, we have a multivariate point process $N(t)=$ $\left(N_{1}(t), \ldots, N_{K}(t)\right)^{\prime}$ such that its conditional intensity function satisfies

$\lim _{h \backslash 0} \frac{1}{h} \operatorname{Prob}\left\{A\right.$ event $\left.\operatorname{in}(t, t+h] \mid H_{t}\right\}$

$=\mu_{A}+\sum_{C=1}^{K} \sum_{\sigma_{C, j} \leq t} \gamma_{A C}\left(t-\sigma_{C, j}\right)$,

where $\sigma_{C, j}$ are the times of events of process $C$ and $\gamma_{A C}(u)$ are some nonnegative, integrable link functions which vanish for $u<0$. The constant $\mu_{A}$ here characterizes the spontaneous activity of process $N_{A}$ while $\gamma_{A C}\left(t-\sigma_{C, j}\right) \cdot h$ can be interpreted as the amount by which the probability for the occurrence of an $A$ event in $(t, t+h]$ is increased by the $C$ events at time $\sigma_{C, j}$. The restriction $\gamma_{A C}(u)=0$ for $u<0$ expresses the fact that only events from the past can have an influence. If the link function $\gamma_{A C}(u)$ is zero for all $u$, which we denote by $\gamma_{A C}(\cdot) \not \equiv 0$, the process $N_{C}$ does not influence process $N_{A}$ at all. Apart from the fact that such processes do not allow for the modeling of refractory periods (e.g. by setting the probability for a new $A$ event to zero directly after an $A$ event has occurred), this seems to be a reasonable model for synaptic interactions. 
Table 1

Estimated delays in milliseconds derived from the ordinary phase curves with $95 \%$ confidence intervals for the Hawkes model data

\begin{tabular}{|c|c|c|c|c|c|}
\hline$\hat{u}_{A B}$ & 0 & 1 & 2 & 3 & 4 \\
\hline 1 & $21.3 \pm 1.0$ & & & & \\
\hline 2 & $39.9 \pm 7.0$ & $21.4 \pm 1.2$ & & & \\
\hline 4 & $48.2 \pm 6.7$ & $40.4 \pm 6.6$ & $21.5 \pm 0.7$ & $21.8 \pm 1.8$ & \\
\hline 5 & $60.8 \pm 7.8$ & $42.9 \pm 3.1$ & $21.4 \pm 6.9$ & $21.4 \pm 1.1$ & $21.0 \pm 1.8$ \\
\hline
\end{tabular}

Following our intuition we denote as the real graph the vertices $\{1, \ldots, K\}$ with the (directed) edge $(B, A)$ present if and only if $\gamma_{A B}(\cdot) \not \equiv 0$ as a function. In this case we say that $B$ is a parent of $A$ and $A$ is a child of $B$.

We now investigate the relations between the real and the conditional independence graph as defined in the last section for this model. We start by investigating the properties of the inverse spectral matrix. For this, let

$$
G_{A C}(\lambda)=\int_{-\infty}^{\infty} \gamma_{A C}(u) \exp (-i \lambda u) \mathrm{d} u
$$

be the Fourier transform of the link function $\gamma_{A \mathrm{C}}(u)$ and $G(\lambda)$ be the matrix with entries $G_{A C}(\lambda)$. Hawkes (1971b) has shown that the spectral matrix of the point process is given by

$f(\lambda)=\frac{1}{2 \pi}\{I-G(\lambda)\}^{-1} D\left\{I-G(-\lambda)^{\prime}\right\}^{-1}$,

where $D$ is the diagonal matrix with the mean intensities $p_{1}, \ldots, p_{K}$ in the diagonal. As a consequence, we obtain

$g(\lambda)=f^{-1}(\lambda)=2 \pi\left\{I-G(-\lambda)^{\prime}\right\} D^{-1}\{I-G(\lambda)\}$,

which yields for $A \neq B$

$$
\begin{aligned}
g_{A B}(\lambda)= & -\frac{2 \pi}{p A} G_{A B}(\lambda)-\frac{2 \pi}{p B} G_{B A}(-\lambda) \\
& +\sum_{C=1}^{K} \frac{2 \pi}{p C} G_{C A}(-\lambda) G_{C B}(\lambda) .
\end{aligned}
$$

As noted in the last section we have an edge between $A$ and $B$ in the conditional correlation graph if and only if $g_{A B}(\cdot) \not \equiv 0$. It can now easily be seen from (Eq. (6))

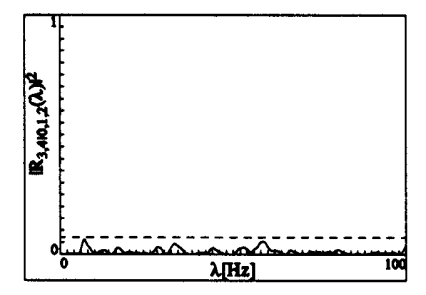

Fig. 6. Estimated partial coherence $\left|R_{3,4 \mid 0,1,2}(\lambda)\right|^{2}$ for the Hawkes model data without process $N_{5}$. that this does not imply $G_{A B}(\cdot) \not \equiv 0$ or $G_{B A}(\cdot) \not \equiv 0$, and vice versa. Thus, the correlation graph and the real graph do not coincide in general nor is it obvious how the real graph can be deduced from the correlation graph.

In particular, it may happen that $g_{A B}(\cdot) \not \equiv 0$ while both $G_{A B}(\cdot)$ and $G_{B A}(\cdot)$ are zero. This occurs if $N_{A}$ and $N_{B}$ both influence a third process $N_{C}$, that is if $G_{C A}(\cdot) \not \equiv 0$ and $G_{C B}(\cdot) \not \equiv 0$ ( $A$ and $B$ are parents of a joint child $C$ ). This effect, which seems to be strange from a first point of view, is, however, quite natural. To see this, we consider independent point processes $N_{1}$, $N_{2}$ and $\varepsilon$ and their superposition $N_{3}(t)=N_{1}(t)+$ $N_{2}(t)+\varepsilon(t)$. Then $N_{1}$ and $N_{2}$ are no longer independent conditional on the process $N_{3}$ and the corresponding conditional correlation graph would show a connection between $N_{1}$ and $N_{2}$.

This effect is well known for graphical models. It is called marrying parents of a joint child and the resulting undirected conditional correlation graph is called moral graph (Whittaker, 1990, Section 3.9). Formally we define the moral graph of a directed graph as the undirected graph containing the edge $(A, B)$ if either $(A, B)$ or $(B, A)$ is an edge in the directed graph or if $A$ and $B$ are parents of a joint child $C$, i.e. $(A, C)$ and $(B, C)$ are edges of the directed graph for some $C$.

Theoretically, it may also happen that an edge of the real graph is lost in the conditional correlation graph (i.e. $g_{A B}(\cdot) \equiv 0$ while $G_{A B}(\cdot) \not \equiv 0$ or $G_{B A}(\cdot) \not \equiv 0$ ). However, this requires a very specific functional relation of the link functions which hardly occurs in practice.

Despite the problems discussed above, we are able to obtain the real graph from the correlation graph in a large number of situations. To see this, we consider an acyclic directed graph which consists of a completely ordered set of vertices, that is

1. $A<B$ or $B<A$ for all $A, B$ with $A \neq B$,

2. $A<A$ does not hold,

3. $A<B$ and $B<C$ implies $A<C$,

and satisfies the restriction that a component of the point process may only excite its successors, that is the link function $\gamma_{A B}(\cdot)$ and thus $G_{A B}(\cdot)$ vanish for $A=B$ and $A<B$. Note that the assumption of a complete ordering is equivalent to the fact that there exist no directed cycles (Whittaker, 1990, Lemma 3.5.1). With- 
Table 2

Estimated delays in milliseconds derived from the partial phase curves with $95 \%$ confidence intervals for the Hawkes model data

\begin{tabular}{lllllll}
\hline$\hat{u}_{1,0}$ & $\hat{u}_{2,0}$ & $\hat{u}_{2,1}$ & $\hat{u}_{3,1}$ & $\hat{u}_{4,2}$ & $\hat{u}_{5,3}$ & $\hat{u}_{5,4}$ \\
\hline $20.7 \pm 1.1$ & $21.3 \pm 1.7$ & $20.8 \pm 1.3$ & $20.1 \pm 0.8$ & $21.1 \pm 1.1$ & $21.2 \pm 0.9$ & $20.8 \pm 1.5$ \\
\hline
\end{tabular}

out loss of generality we can assume that the ordering is the ordering of natural numbers which implies that the matrix $G(\lambda)$ is zero on and above the diagonal for acyclic directed graphs after same reordering. Examples of such acyclic directed graphs are the graphs in Fig. 3.1(a) and Fig. 3.5(a).

We obtain for $A>B$ from (Eq. (6))

$g_{A B}(\lambda)=-\frac{2 \pi}{p_{A}} G_{A B}(\lambda)+\sum_{C=A+1}^{K} \frac{2 \pi}{p_{C}} G_{C A}(-\lambda) G_{C B}(\lambda)$

Now if $A$ and $B$ are not parents of a joint child, the second term in (Eq. (7)) vanishes and we have $g_{A B}(\cdot) \equiv 0$ if and only if $G_{A B}(\cdot) \equiv 0$.

In the complementary case where $A$ and $B$ have a joint child we can show under additional assumptions, e.g. for the common exponential link functions

$\gamma_{A B}(u)=\alpha_{A B} \exp \left(-\beta_{A B}\left(u-u_{A B}\right)\right)$,

that $g_{A B}(\cdot) \not \equiv 0$ (Appendix A), which corresponds to the marrying parents effect. Thus we have proved the following

Theorem: Suppose the real graph of the point process model (Eq. (5)) is acyclic and the link functions are exponential as in (Eq. (8)). Then the conditional correlation graph coincides with the moral graph of the point process.

Further one can show for an acyclic real graph and exponential link functions that if $A$ and $B$ are not parents of a joint child the partial phase spectrum fulfills

$$
\left.\frac{\partial \phi_{A B \mid C_{A B}}(\lambda)}{\partial \lambda}<0 \text { for all } \lambda \text { if } G_{A B}(\cdot) \not \equiv 0 \quad \text { (i.e. } A>B\right)
$$

and

$\frac{\partial \phi_{A B \mid C_{A B}}(\lambda)}{\partial \lambda}>0$ for all $\lambda$ if $G_{B A}(\cdot) \not \equiv 0 \quad$ (i.e. $\left.A<B\right)$.

For joint parents no assertion of this form is possible.

These results imply that an acyclic directed graph of a model (Eq. (5)) with exponential link functions can completely be identified (including the ordering of the vertices) by the following

\subsubsection{Identification procedure}

(1) Using the partial coherences, we draw the conditional correlation graph. For data, the test procedure derived in Section 2.6 has to be used.

(2) We identify the direction of those connections for which either Eq. (9) or Eq. (10) is fulfilled (here for data a corresponding test procedure still has to be developed - however, compare the examples in Section 3) and which cannot be affected by a marrying parents effect.

(3) If $A$ and $B$ are parents of a joint child we calculate the conditional correlation graph for the reduced graph obtained by deleting all successors of $A$ and $B$. It is obvious from (Eq. (7)) that in this reduced correlation graph an edge between $A$ and $B$ is present if and only if it is present in the real graph. Thus, we can delete those edges which are due to a marrying parents effect. The phase spectrum between $A$ and $B$ in the reduced correlation graph gives the direction.

Examples for the use of this identification procedure are given in Section 3. The steps (ii) and (iii) may be more complicated than it seems since an edge between $A$ and $B$ may not only result from a marrying parents effect if there are edges $(A, C)$ and $(B, C)$ which are directed towards $C$, but also if one of the edges $(A, C)$ and $(B, C)$ itself is affected by a marrying parents effect and its direction therefore is unknown.

For example in Fig. 2, the edge $(B, C)$ may be due to a marrying parents effect. Since its existence and also its direction is not clear, the edge $(A, C)$ may also be due to a marrying parents effect. In (iii) one tests the edge between $B$ and $C$ with the conditional correlation graph of $A, B$ and $C$ and then (if necessary) the edge between $A$ and $B$ with the conditional correlation of $A$ and $B$ alone (that is by the coherence between $A$ and $B)$. Testing first $A$ and $B$ would lead to the same result. However, in order to get a unique result also in the data-dependent case we suggest to start with the edges with the highest order with respect to the ordering of the vertices (i.e. with $(B, C)$ in the above example).

\subsection{Testing for a connection in the conditional correlation graph}

An edge in the correlation graph is defined to be missing if the corresponding partial spectral coherence is zero at all frequencies. However if concerned with data, we have to use the empirical partial coherence (calculated as described at the end of Section 2.2), 
(a)

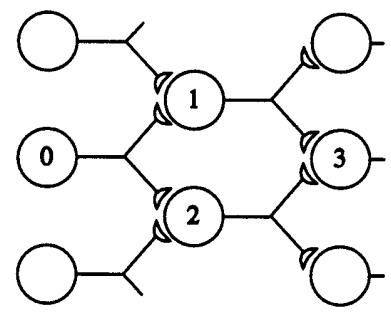

(b)

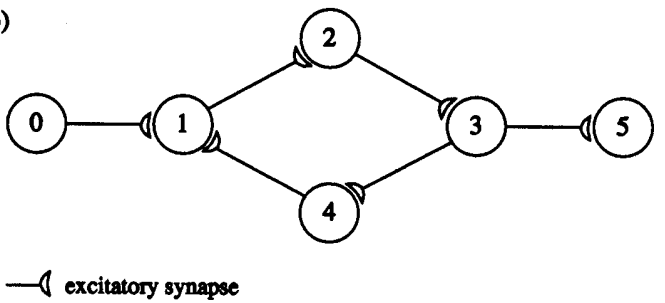

Fig. 7. Neural networks with (a) diverging and converging synaptic connections and (b) an excitatory feedback loop.

which in this case will only be approximately zero. In order to find a test for an edge, we therefore have to construct a threshold for the empirical partial spectral coherence. If the estimate $\left|\hat{R}_{A B \mid C_{A B}}(\lambda)\right|^{2}$ exceeds that threshold for at least one frequency, we decide that there is an edge between $A$ and $B$.

As a threshold, we take the upper $\alpha$-quantile of the asymptotic distribution of the maximum of $\left|\hat{R}_{A B \mid C_{A B}}(\lambda)\right|^{2}$ over all frequencies under the assumption that the two processes $N_{A}$ and $N_{B}$ are uncorrelated after removing the linear effects of the process $N_{C_{A B}}$. Then the probability of $\left|\hat{R}_{A B \mid C_{A B}}(\lambda)\right|^{2}$ exceeding this threshold for at least one frequency is $\alpha$ if there is no edge between $A$ and $B$.

In order to determine this threshold, we first note that under the assumption of $R_{A B \mid C}(\lambda)=0$ the estimate $\left|\hat{R}_{A B \mid C_{A B}}(\lambda)\right|^{2}$ has the same limiting distribution as $\frac{\left|\hat{f}_{A B \mid C_{A B}}(\lambda)\right|^{2}}{f_{A A \mid C_{A B}}(\lambda) f_{B B \mid C_{A B}}(\lambda)}$.

Since $\hat{f}_{A B \mid C_{A B}}(\lambda)$ is a differentiable function of the spectral density estimates $\hat{f}_{C D}(\lambda)$ with $C, D=1, \ldots, K$, which are asymptotically normal (Brillinger, 1972, Theorem 4.3), it can be shown by application of the delta-method that the real and imaginary part of

$\sqrt{T}\left(\mid \hat{R}_{A B \mid C_{A B}}(\lambda)-R_{A B \mid C_{A B}}(\lambda)\right)$

are asymptotically independent and normally distributed with mean zero and variance $\sigma_{T}=\pi\left(H_{4} / H_{2}^{2}\right)$ j $w_{T}(\alpha)^{2} \mathrm{~d} \alpha$, where $H_{k}=\int_{0}^{1} h(x)^{k} \mathrm{~d} x$. Therefore, still under the assumption of $R_{A B \mid C_{A B}}(\lambda)=0$,

$\frac{T}{\sigma_{T}}\left|\hat{R}_{A B \mid C_{A B}}(\lambda)\right|^{2}$

is asymptotically $\chi_{2}^{2}$-distributed. Instead of the above approximation, a normal distribution as an approximation of

$\tan h^{-1}\left(\left|\hat{R}_{A B \mid C_{A B}}(\lambda)\right|^{2}\right)-\tan h^{-1}\left(\left|R_{A B \mid C_{A B}}(\lambda)\right|^{2}\right)$

has been used frequently (Brillinger, 1981; Rosenberg et al., 1989). However, in the case $R_{A B \mid C_{A B}}(\lambda)=0$ it seems not to be adequate to approximate the positive distribution of $\tan h^{-1}\left(\left|\hat{R}_{A B \mid C_{A B}}(\lambda)\right|^{2}\right)$ by a normal distribution centered around zero.
Next, we note that the products $\mathrm{d}^{T}(\lambda) \mathrm{d}^{T}(-\lambda)$ and $\mathrm{d}^{T}(\mu) \mathrm{d}^{T}(-\mu)$ are approximately independent for large $T$. Consequently, the estimates $\hat{f}_{A B}(\lambda)$ and $\hat{f}_{A B}(\mu)$ are also approximately independent if the values $\lambda$ and $\mu$ are separated widely enough such that the corresponding smoothing intervals are non-overlapping. The same holds then for the estimates $\left|\hat{R}_{A B \mid C_{A B}}(\lambda)\right|^{2}$ and $\left|\hat{R}_{A B \mid C_{A B}}(\mu)\right|^{2}$. This suggests to consider the maximum of $n_{T}$ independent $\chi_{2}^{2}$-distributed random variables, where $n_{T}$ is the maximum number of frequencies $\lambda$ such that the smoothing intervals of the spectral estimates do not overlap. For the significance level $\alpha$, we obtain from this as a simultaneous upper $(1-\alpha)$-test bound

$\frac{\sigma_{T}}{T} \chi_{2,(1-\alpha)^{1 / n} T}^{2}$

where $\chi_{2, p}^{2}$ denotes the $p$-quantile of the $\chi_{2}^{2}$-distribution. Strictly speaking this is only a threshold for the maximum of $\left|\hat{R}_{A B \mid C_{A B}}(\lambda)\right|^{2}$ at those $n_{T}$ frequencies where the spectral estimates do not overlap. It seems to be very difficult to obtain an exact threshold for the maximum at all frequencies (such a derivation requires a stochastic process approximation, Bickel and Rosenblatt (1973)). Furthermore, we expect only minor changes from such an improvement.

\section{Simulations}

In this section we investigate the properties of the proposed method - both for a Hawkes model and several neural ensembles.

We start with the ideal situation of a Hawkes model (Eq. (5)) with exponential link functions (Eq. (8)) and the acyclic graph of Fig. 3(a). In our simulation, we have used connections with different transmission rates which are determined by the ratio $\alpha_{A B} / \beta_{A B}$. Further, the parameters $\beta_{A B}$ have been chosen such that the influence of a single event of process $N_{B}$ on process $N_{A}$ dies away quickly compared with the time delay $u_{A B}$.

Figs. 4 and 5 illustrate the differences between ordinary coherences and phase-spectra on the one side and partial coherences and partial phase-spectra on the other side. The partial spectral coherences have been calculated as described at the end of Section 2.2. The 


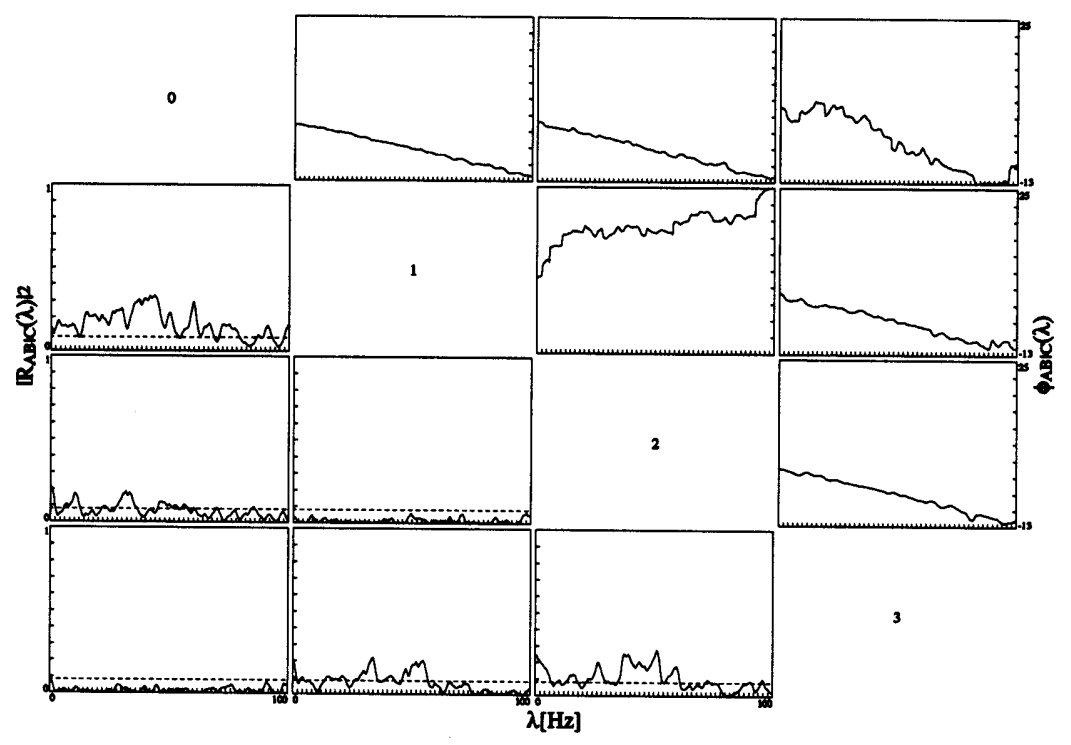

Fig. 8. Estimated partial coherences $\left|R_{A B \mid C_{A B}}(\lambda)\right|^{2}$ and partial phase spectra $\phi_{A B \mid C_{A B}}(\lambda)$ for the data simulated from the network in Fig. 7(a). The horizontal dashed lines represent a $95 \%$ test bound for the supremum of all partial coherences under the assumption that the two processes are uncorrelated conditional on the other processes.

horizontal dashed line is the $95 \%$ test bound for the maximum of the ordinates as described in Section 2.6, which means that an edge is present if the partial spectral coherence exceeds this bound at one or more frequencies.

The coherences in Fig. 4 show that all six processes are pairwise coupled. Further, the linear pattern of the phase curves indicates that the connections are dominated by a time delay. Table 1 gives the estimated delays which have been computed using a weighted least squares algorithm (Rosenberg et al., 1989). Here, the estimated delay of $39.9 \pm 7.0 \mathrm{~ms}$ between processes $N_{0}$ and $N_{2}$ is particularly interesting because it corresponds not to the weaker direct connection but to the indirect connection via $N_{1}$. Altogether, neither coherences nor phase-spectra provide much information about the structure of the network in Fig. 3.

A much better understanding of the connectivity in the network is provided by the partial coherences (Fig. 5), which vanish in most cases where two processes are not directly connected while they differ significantly from zero elsewhere. For our analysis of the net, we follow the identification procedure in Section 2.5. Fig. 3 (b) shows the conditional correlation graph as it follows from the estimated partial coherences. We have added directions for those edges where the phase spectrum nearly follows a straight line and which are not potentially affected by a marrying parents effect. It is already clear from this graph that the real graph is acyclic. The edges between 0 and 1 and between 3 and 4 may be due to the marrying parents effect, which has to be investigated in the corresponding reduced graphs with all successors deleted. Thus, we have to test $\left|\hat{R}_{3,4 \mid 0,1,2}(\cdot)\right|^{2}$ and $\left|\hat{R}_{0,1}(\cdot)\right|^{2}$ separately.
First, Fig. 6 implies that the connection between 3 and 4 is not significant and that the edge in the conditional correlation graph is solely due to the marrying parents effect. Next, Fig. 4 shows that $\left|\hat{R}_{0,1}(\cdot)\right|^{2}$ is significantly different from zero. From the corresponding phase-curve $\hat{\phi}_{0,1}(\cdot)$, which is approximately linear, it finally can be seen that the connection is directed from 0 to 1 .

The linear partial phases now yield estimates for the delays of the direct connections which have been identified. The estimates given in Table 2 show that all connections have about the same delay of $21 \pm 1 \mathrm{~ms}$. In the case of processes $N_{0}$ and $N_{2}$, this estimate differs significantly from that derived from the ordinary phase. This together with the substantial reduction of the partial coherence suggests that the indirect connection via $N_{1}$ has a predominant effect on the timing relation between the two processes.

Although we have plotted all phase curves in this paper for illustration, it usually makes no sense to plot phase curves associated with non-significant (partial) coherences. If the coherence is zero, the corresponding phase is not defined at all and the estimate has an infinite variance (Brillinger, 1981, Eq. (8.7.15)). In these cases, no conclusions can be derived from the estimate even though the actual phase curves in Fig. 5 look smooth due to the estimation method. Caution is necessary especially if the (partial) coherence is significant only for some frequencies (as for the inhibitory connections in Fig. 12. In this situation, a visual inspection of the phase curves over all frequencies may be misleading as only part of the values should be used for the determination of the time delay (which is automatically done by the weighted least squares method of Rosenberg et al. (1989)). 


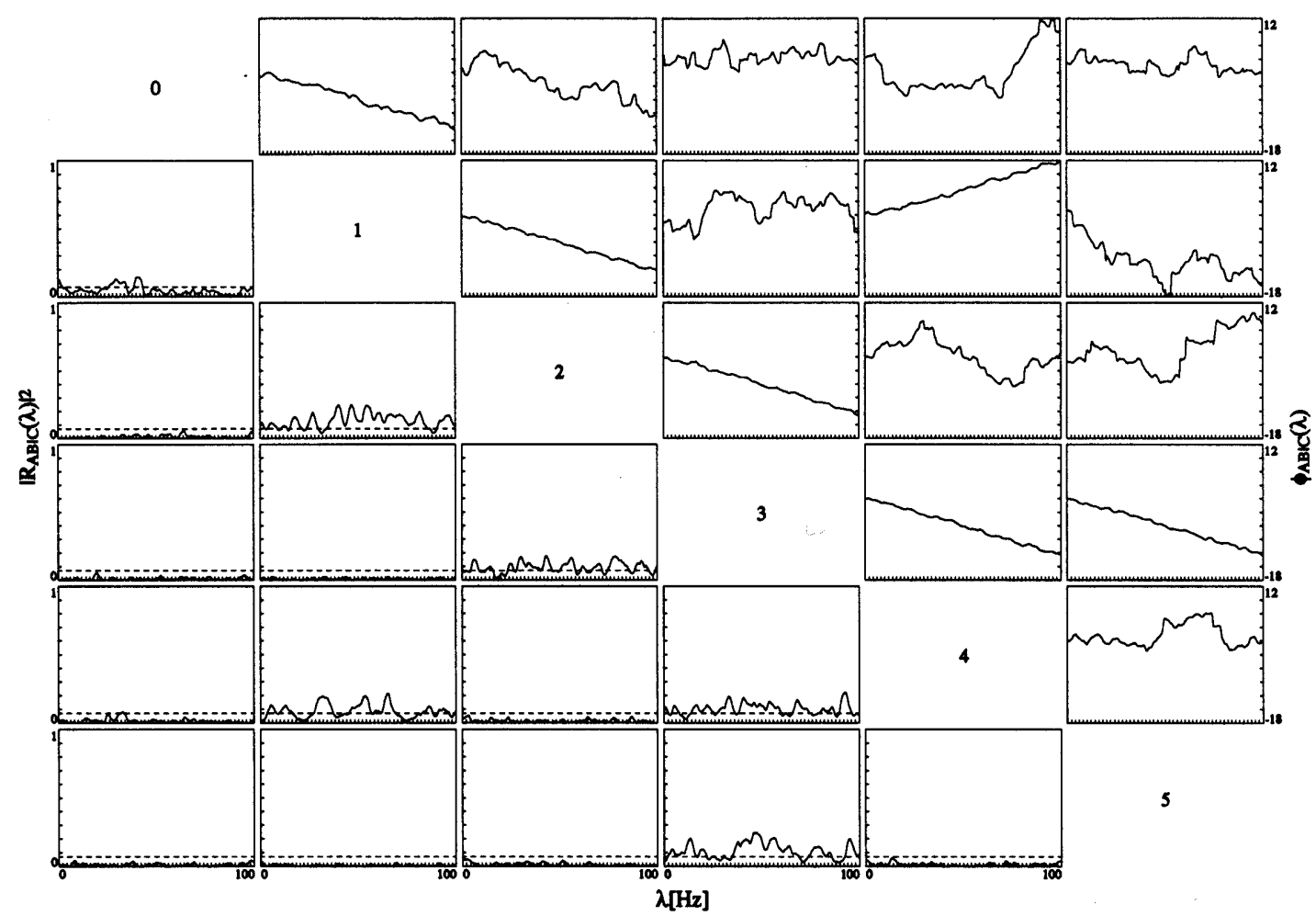

Fig. 9. Estimated partial coherences $\left|R_{A B \mid C_{A B}}(\lambda)\right|^{2}$ and partial phase spectra $\phi_{A B \mid C_{A B}}(\lambda)$ for the data simulated from the network in Fig. 7(b). The horizontal dashed lines represent a $95 \%$ test bound for the supremum of all partial coherences under the assumption that the two processes are uncorrelated conditional on the other processes.

We now turn to the discussion of examples of neural spike trains which have been generated by the neural network simulator GENESIS Version 1.4 on the basis of the Hodgkin-Huxley ion channel model (Bower and Beeman, 1995). The simulated networks were built of simple nerve cells whose dynamics were derived from measurements of the squid giant axon. In each nerve cell, the leakage current was disturbed by random injections of small currents in order to model the stochastic variations of the somatic membrane potential. As neural networks typically consist of a large number of neurons, the nerve cells were additionally excited by Poisson point processes which represented the stimulation due to further neurons whose spike trains have not been recorded. Without some stochasticity of this kind in the data, the identification method would fail to distinguish direct from indirect connections since in purely deterministic systems each indirect connection has the same effect as a direct connection with cumulated time delay. On the other hand, if the system is disturbed by too much noise, the connections do not transmit enough action potentials to be identified.

Again we use the identification method from Section 2.5. Several problems may arise. In particular, it is not clear whether the method works at all due to non-linear dynamics in spike trains (for example due to refractory periods). Problems may also arise if the graph is not acyclic or if connections are inhibitory which would correspond to negative link functions in the Hawkes model. However, the following simulations show that the proposed method works well despite these problems.

Fig. 7(a) illustrates part of a neural network with diverging and converging synaptic connections. Divergence and convergence of neural connections are common principles in the organization of the nervous system. Fig. 8 shows the partial coherences and partial phase-spectra between the spike trains recorded from neurons 0 to 3 . The partial coherences correctly identify the four connections and the conditional correlation graph therefore is identical to the graph. It is interesting that the marrying parents effect does not cause an edge between 1 and 2 . Theoretically, the marrying parents effect may also hide a connection between 1 and 2 which, however, can be excluded by looking at the reduced graph, that is by testing $\left|\hat{R}_{1,2 \mid 0}(\cdot)\right|^{2}$, which indeed stays below the threshold (not shown in a figure). Thus, the graph has been correctly identified. The partial phase curves now yield estimates for the delays of about $19 \pm 1.5 \mathrm{~ms}$.

In the next example, the data-set has been simulated from a network of six neurons with an excitatory feedback connection (Fig. 7(b)). Here, brief excitatory 

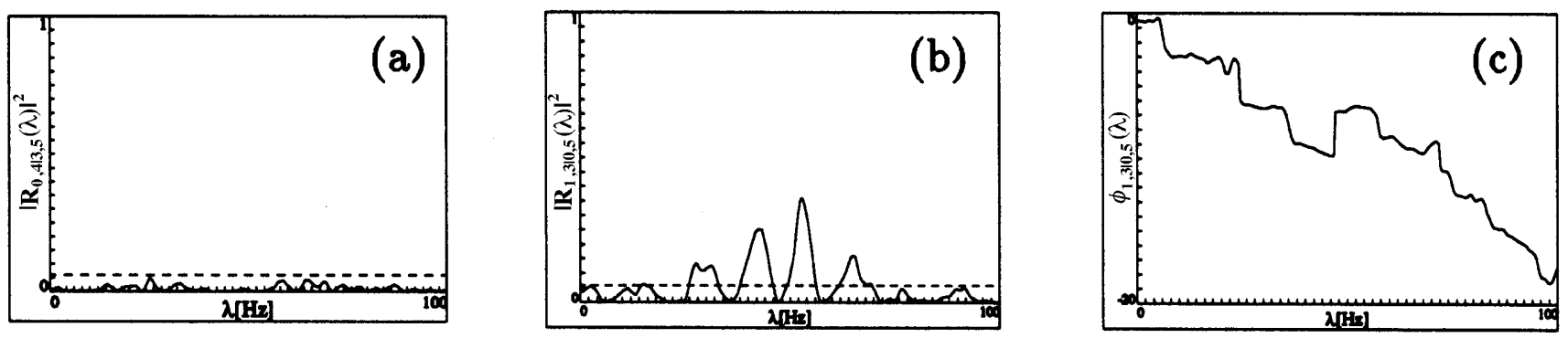

Fig. 10. (a) Estimated partial coherences $\left|R_{04 \mid 3,5}(\lambda)\right|^{2}$ and (b) $\left|R_{1,3 \mid 0,5}(\lambda)\right|^{2}$ and (c) estimated partial phase $\phi_{1,3 \mid 0,5}(\lambda)$ for the network in Fig. $7($ b).

input can produce long-lasting neural activity within the closed loop through circulation of activity among the neurons. Therefore, such reverberating circuits have been used as an explanatory model for the short-term memory. The graph is not acyclic and therefore the assumptions underlying the results of Section 2.5 are violated.

Fig. 9 demonstrates that the structure of the network can be identified completely by the application of the partial coherences and the partial spectra. A marrying parents effect might occur between 0 and 4 (in fact the partial coherence shows a tiny peak above the threshold, whose significance, however, seems questionable). Since the graph contains a cycle it is not possible to investigate the reduced graph obtained by deleting the joint child 1 and its successors (since 4 itself is a successor). A reasonable procedure in this situation seems to delete the child 1 and all successors until one reaches an immediate predecessor of the parents and to investigate whether this leads to an 'adequate' reduction of the partial coherence (what is 'adequate' clearly needs further theoretical investigation). In the present context, this means deleting 1 and 2 . The resulting $\left|\hat{R}_{0,4 \mid 3,5}(\cdot)\right|^{2}$ remains below the threshold (Fig. 10(a)) which is a clear indication that there is no connection between 0 and 4 . Thus, the graph has been identified correctly. The estimated delays again are about $19 \pm 1.5$ ms.

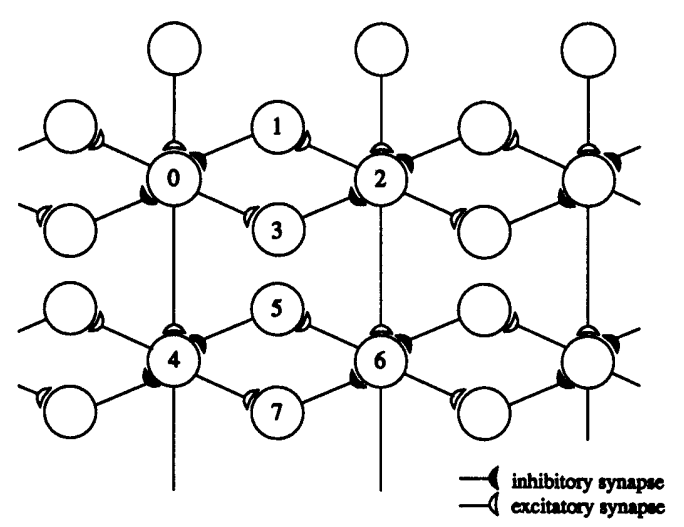

Fig. 11. Neural network with lateral inhibition.
Now suppose that we have recorded spike trains only from neurons $0,1,3$, and 5 . In the reduced network, neurons 1 and 3 are connected by two direct connections which have opposite directions. As described in Section 2.3, we cannot resolve the full structure of the network in this situation, which is demonstrated by Fig. 10. Here, the partial coherence $\left|\hat{R}_{1,3 \mid 0,5}(\cdot)\right|^{2}$ (Fig. 10(b)) correctly detects that the two neurons are directly connected. The slope of the corresponding partial phase curve (Fig. 10(c)) now indicates that impulses are transmitted in the direction of neuron 3, which corresponds to the main pathway in the network. But the partial phase does not provide any information about the presence of an excitatory feedback connection, and thus we are left with an incomplete identification of the network.

In the last example, we use the presented methods for the identification of a neural network that includes also inhibitory synaptic connections. Fig. 11 shows part of the simulated network which consisted of more than 30 neurons. This arrangement of excitatory and inhibitory connections produces lateral inhibition, which is quite common in sensory pathways to facilitate localization and sharpen contrast. The data-set to be analyzed consists of eight spike trains which have been recorded from neurons 0 to 7 (Fig. 11). The estimated partial coherences and partial phase-spectra for this data-set are given in Fig. 12. Comparing the results with the structure of the network, we notice a striking difference between excitatory and inhibitory connections. The six excitatory connections are easy to detect since the partial coherences stay well above the threshold and the partial phase curves show clearly a linear pattern as in the examples before, whereas in the case of inhibitory connections the partial coherences exhibit smaller peaks at low frequencies and vanish for frequencies above 25-30 Hz. Consequently, the corresponding partial phase-spectra are approximately linear only over a small range of frequencies, which makes it difficult to estimate the delay for an inhibitory connection. Nevertheless, the direction of the inhibitory connections are identified correctly if one uses the weighted least squares procedure of Rosenberg et al. (1989) which automatically restricts in the present case to low fre- 


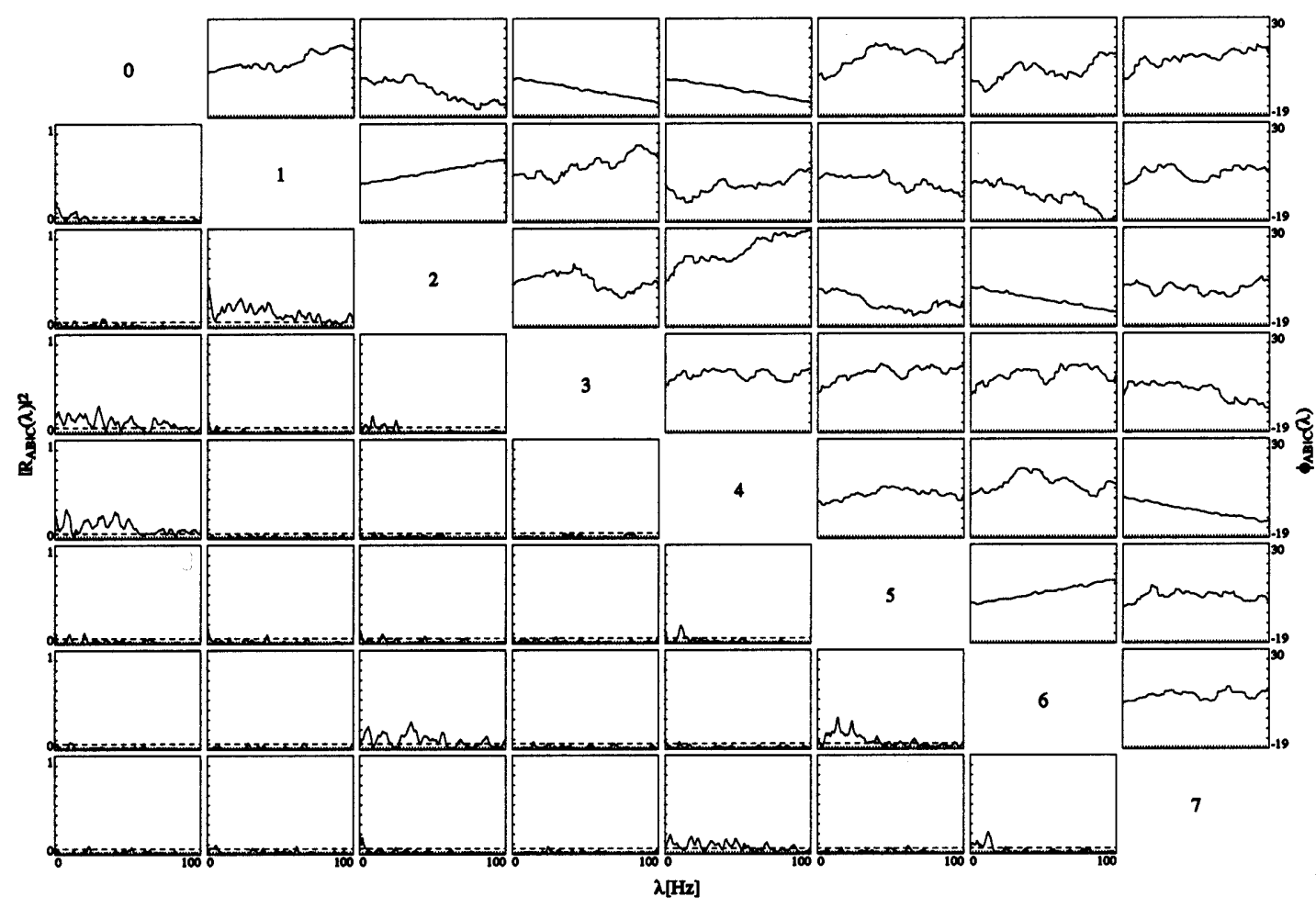

Fig. 12. Estimated partial coherences $\left|R_{A B \mid C_{A B}}(\lambda)\right|^{2}$ and partial phase spectra $\phi_{A B \mid C_{A B}}(\lambda)$ for the data simulated from the network in Fig. 11. The horizontal dashed lines represent a $95 \%$ test bound for the supremum of all partial coherences under the assumption that the two processes are uncorrelated conditional on the other processes.

quencies. Further, we note that the partial coherences between spike trains which are not directly linked do not necessarily remain subthreshold for all frequencies. For connection $(0,5)$ and $(2,7)$ this again is a marrying parents effect which can be excluded by calculating the partial coherences $\left|\hat{R}_{0,5 \mid 1,2,3,6}(\cdot)\right|^{2}$ and $\left|\hat{R}_{2,7 \mid 0,1,3,4}(\cdot)\right|^{2}$. Apart from some questionable connections (e.g. $(0,2)$ ), the method therefore successfully identifies the graph. However, it is clear that it is difficult to distinguish partial coherences of inhibitory connections from partial coherences where no connection is present (see Fig. 13).

\section{Concluding remarks}

In this paper, we have presented a conditional correlation graph for the dependence structure of multivariate point process. Based on this graph, a method has been suggested to identify synaptic connections in neural ensembles. The method is based on the partial coherence, which has been used earlier by several authors to distinguish between direct and indirect connections. The simulations have demonstrated that the method can successfully be applied in different situations of neural ensembles.

The method has several limitations. One requirement for the method to work is that the signal to noise ratio is neither to high nor to low. This has already been discussed in Section 2.6. Furthermore, the method cannot discriminate between direct connections and indirect connections via unobserved neurons. Another limitation is that the method basically is a linear one while neural activity shows also nonlinear dynamics. It would be interesting to investigate whether the identification problems with the inhibitory connections are due to nonlinear dynamics or to a low signal to noise ratio.

A lot of work remains to be done to investigate the relations between the conditional correlation graph and a more realistic model for neuron firing than the Hawkes model used in Section 2.5 such as the threshold crossing model of Brillinger and Segundo (1979). Furthermore, more simulations and data examples are necessary to study the limitations of the method. Here, several specific situations need to be addressed, such as inhibitory connections or the modulating effects of external (periodic) stimulation. In addition, several specific problems have to be solved, such as the question of a test for a direction based on the partial phase spectrum or the identification procedure in cyclic graphs.

\section{Acknowledgements}

This work has been supported by grants from the Ministerium für Wissenschaft und Forschung Baden- 

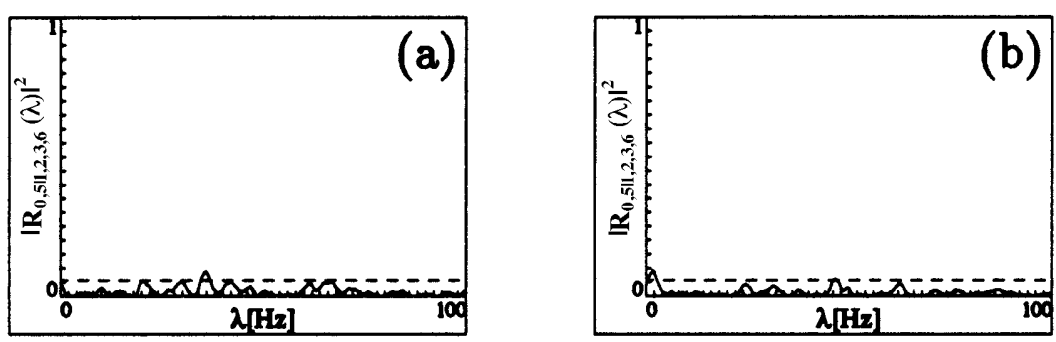

Fig. 13. (a) Estimated partial coherences $\left|R_{0,5 \mid 1,2,3,6}(\lambda)\right|^{2}$ and (b) $\left|R_{0,5 \mid 1,2,3,6}(\lambda)\right|^{2}$ for the network in Fig. 11.

Württemberg, the Deutsche Forschungsgemeinschaft (SA 435) and a European Union Human Capital and Mobility Programme (ERB CHRX-CT 940693). We are grateful to J. Noll, who has done the simulations with GENESIS, and to two anonymous referees whose comments led to a substantial improvement of the paper. After submitting this present paper it came to our attention that Brillinger (1996) has also discussed graphical models for time series and point processes based on the partial spectral coherence. Apart from the basic definition of the correlation graph there is nearly no overlap with the present paper.

\section{Appendix A}

First, we prove that under the assumption of the theorem in Section $2.5 g_{A B}(\cdot)$ differs from zero whenever $A$ and $B$ are parents of a joint child. For this, we observe that since $\gamma_{A B}(\cdot)$ is nonnegative we have $G_{A^{\prime} B^{\prime}}(0)>0$ if and only if $\gamma_{A^{\prime} B^{\prime}}(\cdot) \not \equiv 0$. Now if $G_{A B}(\cdot) \equiv$ 0 then by (Eq. (7)) $g_{A B}(0)>0$. On the other hand, if $G_{A B}(\cdot) \not \equiv 0$ and $A$ and $B$ have one joint child $C$ then with

$G_{A B}(\lambda)=\mathrm{e}^{-i \lambda u_{A B}} \frac{\alpha_{A B}}{\beta_{A B}+i \lambda}$

(Hawkes, 1971a) $g_{A B}(\lambda)=0$ would imply

$p_{C} \alpha_{A B}\left(\beta_{C A}-i \lambda\right)\left(\beta_{C B}+i \lambda\right) \mathrm{e}^{-i \lambda u_{A B}}$

$=p_{A} \alpha_{C A} \alpha_{C B}\left(\beta_{A B}+i \lambda\right) \mathrm{e}^{-i \lambda\left(u_{C A}+u_{C B}\right)}$,

which holds if and only if $\alpha_{A B}=0$ which contradicts $G_{A B}(\cdot) \not \equiv 0$. Therefore, it must be $g_{A B}(\lambda) \neq 0$.

Next, we prove (Eqs. (9) and (10)). If $A$ and $B$ are not parents of a joint child and $\gamma_{A B}(\cdot) \not \equiv 0$, we obtain for the partial phase spectrum

$\phi_{A B \mid C_{A B}}(\lambda)=\arg G_{A B}(\lambda)=-\lambda u_{A B}-\arctan \left(-\lambda / \beta_{A B}\right)$

and thus

$\frac{\mathrm{d} \phi_{A B \mid C_{A B}}(\lambda)}{\mathrm{d} \lambda}=-u_{A B}-\frac{\beta_{A B}}{\beta_{A B}^{2}+\lambda^{2}}<0$.

The case $\gamma_{B A}(\cdot) \not \equiv 0$ is similar.
The software used for the computation of partial coherences and partial phase-spectra has been implemented in $C$ on an UNIX-system. Enquiries about the software should be sent to eichler@statlab.uni-heidelberg.de. The simulation software GENESIS is available via anonymous FTP at babel.caltech.edu.

\section{References}

Alloway KD, Johnson MJ, Wallace MB. Thalamocortical interactions in the somatosensory system: Interpretation of latency and cross-correlation analyses. J Neurophysiol 1993;70:892-908.

Bickel PJ, Rosenblatt M. On some global measures of the deviations of density function estimates. Ann Stat 1973;1:1071-95.

Bliss TVP, Collingridge GL. A synaptic model of memory: Longterm potentiation in the hippocompus. Nature 1993;361:31-9.

Bower JM, Beeman D. The Book of GENESIS, New York: Springer, 1995.

Brillinger DR. The spectral analysis of stationary interval functions. Proceedings of the Sixth Berkeley Symposium, Vol 1. California: Berkely, 1972:483-513.

Brillinger DR, Bryant HL, Segundo JP. Identification of synaptic interactions. Biol Cybernet 1976;22:213-29.

Brillinger DR, Segundo JP. Empirical examination of the threshold model for neuron firing. Biol Cybernet 1979;35:213-20.

Brillinger DR. Time Series: Data Analysis and Theory. New York: McGraw Hill, 1981.

Brillinger DR. Remarks concerning graphical models for time series and point processes. Rev Econom 1996;16:1-23.

Cox DR, Wermuth N. Multivariate dependencies. London: Chapman and Hall, 1996.

Dahlhaus R. Graphical interaction models for multivariate time series, Preprint, Universität Heidelberg, 1995

Gersch W. Causality of driving in electrophysiological signal analysis. J Math Biosci 1972;14:177-96.

Gersch W, Goddard G. Epileptic focus location: Spectral analysis method. Science 1970;169:701-2.

Gerstein GL, Aertsen AMHJ. Representation of cooperative firing activity among simultaneously recorded neurons. J Neurophysiol 1985;54:1513-28.

Gray CM, Maldonado PE, Wilson M, McNaughton P. Tetrodes markedly improve the reliability and yield of multiple single-unit isolation from multiunit recordings in cat striate cortex. J Neurosci Methods 1995;63:43-54.

Grinvald A, Frostig RD, Lieke E, Hildesheim R. Optical imaging of neuronal activity. Physiol Rev 1988;68:1285-366.

Hata Y, Tsumoto T, Sato H, Tamura H. Horizontal interactions between visual cortical neurones studied by cross-correlation analysis in the cat. J Physiol (Lond) 1991;441:593-614. 
Hawkes AG. Spectra of some self-exciting and mutually-exciting point process. Biometrika 1971a;58:83-90.

Hawkes AG. Point spectra of some mutually exciting point processes. J R Stat Soc Ser B 1971b;33:438-43.

Kamiński MJ, Blinowska KJ. A new method of the description of the information flow in the brain structures. Biol Cybernet 1991;65:203-10.

Krüger J. Simultaneous individual recordings from many cerebral neurons: Techniques and results. Rev Physiol Biochem Pharmacol 1983;98:176-233.

Lauritzen S. Graphical Models, Oxford: Clarendon Press, 1996.

Lindsay BG, Hernandez YM, Morris KF, Shannon R, Gerstein GL. Dynamic reconfiguration of brain-stem neuronal assemblies-respiratory phase-dependent synchrony versus modulation of firing rates. J Neurophysiol 1992;67:923-30.

Parsons TD, Salzberg BM, Obaid AL, Raccuia-Behling F, Kleinfeld D. Long-term optical recording of patterns of electrical activity in ensembles of cultured neurons. J Neurophysiol 1991;66:316-33.
Rigas AG. Spectral analysis of stationary point processes using fast fourier transform algorithm. J Time Ser Anal 1993;13:441-50.

Rosenberg JR, Amjad AM, Breeze P, Brillinger DR, Halliday DM. The Fourier approach to the identification of functional coupling between neuronal spike trains. Prog Biophys Mol Biol 1989;53:131.

Schuldiner S, Shirvan A, Linial M. Vesicular neurotransmitter transporters: From bacteria to humans. Physiol Rev 1995;75:369-92.

Singer W. Synchronization of cortical activity and its putative role in information processing and learning. Annu Rev Physiol 1993;55:349-74.

Trimble WS, Linial M, Scheller RH. Cellular and molecular biology of the presynaptic nerve terminal. Annu Rev Neurosci 1991;14:93-122.

Whittaker J. Graphical Models in Applied Multivariate Statistics. Chichester: Wiley, 1990.

Wickmann K, Clapham DE. Ion channel regulation by G proteins. Physiol Rev 1995;75:865-85. 